J3eA, Journal sur l'enseignement des sciences et technologies de l'information et des systèmes, Volume 4, Hors-Série 1, 8 (2005)

DOI : http://dx.doi.org/10.1051/bib-j3ea:2005608

(C) EDP Sciences, 2005

Alternateur inertiel à surcharge élevée pour véhicule électrique de combat

M. Martinez et J.-Y. Midy

THALES AES Company

41 boulevard de la République

F-78400 Chatou, France 


\title{
Alternateur inertiel à surcharge élevée pour véhicule électrique de combat
}

\author{
M. Martinez, J.-Y. Midy \\ THALES AES Company - 41bd de la République 78400 CHATOU FRANCE
}

\begin{abstract}
Résumé-La société THALES AES a étudié, sous contrat DGA et en collaboration avec d'autres sociétés, un système de génération électrique capable de délivrer une surcharge importante pendant dix secondes. Cette source d'énergie électrique est destinée à équiper un véhicule de combat à propulsion électrique. La surcharge correspond à un franchissement d'obstacle. Le moteur d'entraînement du générateur n'est supposé délivrer que la puissance permanente tandis que la surcharge dix secondes est prélevée sur l'énergie cinétique des parties tournantes du groupe. Afin de stocker une énergie cinétique importante sans pour autant accroître beaucoup la masse, la vitesse de rotation du générateur est choisie la plus élevée possible tout en étant compatible avec les contraintes mécaniques des parties tournantes. L'inertie tournante qui délivre cette énergie cinétique transitoire doit être suffisante pour que la vitesse de rotation de l'ensemble ne descende pas pendant le transitoire en dessous d'une vitesse égale à la moitié de la vitesse nominale.
\end{abstract}

Ce générateur est aussi un démonstrateur d'alternateur grande vitesse pour applications aéronautiques. Le présent article décrit le système complet retenu, l'inertie tournante, le générateur, le Generator Control Unit (GCU) et les essais réalisés sur une maquette de générateur.

\section{DESCRIPTION GENERALE DU SYSTEME}

Le générateur destiné au véhicule de combat doit délivrer une puissance électrique permanente de $200 \mathrm{~kW}$ sous une tension continue de 540 VDC. Une batterie est branchée en parallèle sur le générateur. Ce dernier doit fournir durant dix secondes une surcharge de $400 \mathrm{~kW}$. Le moteur thermique entraînant l'alternateur est supposé être dimensionné pour cette puissance de $200 \mathrm{~kW}$. La différence entre la surpuissance et la puissance nominale doit être extraite de l'énergie cinétique des parties tournantes.

La vitesse du générateur a été fixée à 40000 tr/min, valeur limite à ce niveau de puissance. La valeur de l'inertie des parties tournantes doit être telle que la vitesse ne descende pas en dessous de
$20000 \mathrm{tr} / \mathrm{min}$ à la fin des dix secondes de surcharge.

Au titre du contrat DGA, la société THALES AES devait réaliser un démonstrateur délivrant un quart de la puissance du générateur préalablement évoqué. Le démonstrateur est destiné à être essayé sur une plate-forme roulante (projet ECCE). Il doit être capable de délivrer une puissance permanente de $50 \mathrm{~kW}$ à $40000 \mathrm{tr} / \mathrm{min}$ et une surcharge de $100 \mathrm{~kW}$ pendant dix secondes entre 40000 et 20000 tr/min. Le générateur du démonstrateur est entraîné par un moteur diesel type DW10 de fourniture PSA, tournant entre 1500 et $4000 \mathrm{tr} / \mathrm{min}$ et délivrant une puissance maximum de $60 \mathrm{~kW}$ à $2000 \mathrm{tr} / \mathrm{min}$. Un multiplicateur de rapport dix a été étudié pour amener la vitesse au niveau des valeurs requises pour le générateur.

Dans le cas du générateur $400 \mathrm{~kW}$ l'énergie cinétique dissipée pendant les dix secondes de surcharge est égale à $2500 \mathrm{~kJ}$ environ et dans le cas $\mathrm{du}$ démonstrateur $625 \mathrm{~kJ}$. Ces fortes valeurs conduisent à des valeurs d'inertie tournante importantes, même à 40000 tr/min : 0.4 kg.m2 dans le premier cas et $0.1 \mathrm{~kg} . \mathrm{m} 2$ dans le deuxième cas. Ces inerties tournantes importantes peuvent créer un effet gyroscopique qui gênera le comportement du véhicule dans un virage.

Pour éviter cet effet gyroscopique on peut concevoir un générateur inertiel à axe de rotation vertical. Une difficulté technologique apparaît alors : le palier butée de l'inertie tournante qui est difficile à réaliser lorsque la vitesse de rotation est de $40000 \mathrm{tr} / \mathrm{min}$. Afin d'éviter cette difficulté une solution a été retenue en collaboration avec le laboratoire L2ES à Belfort (France) : cette solution consiste à créer deux couples gyroscopiques antagonistes qui se neutralisent. Pour cela deux volants d'inertie tournent en sens contraire sur deux arbres distincts. Les effets gyroscopiques sur le véhicule seront complètement éliminés si ces deux couples s'exercent au même point. En réalité les deux volants sont séparés d'une distance qui doit être minimisée.

Cette solution conduit à envisager deux générateurs, chacun étant associé à un volant d'inertie. Le schéma de la fig. 1 montre le montage d'ensemble du groupe qui comporte :

- le moteur thermique accouplé à un 
multiplicateur de rapport dix,

- les deux volants d'inertie et les deux générateurs entraînés par l'intermédiaire d'engrenages coniques.

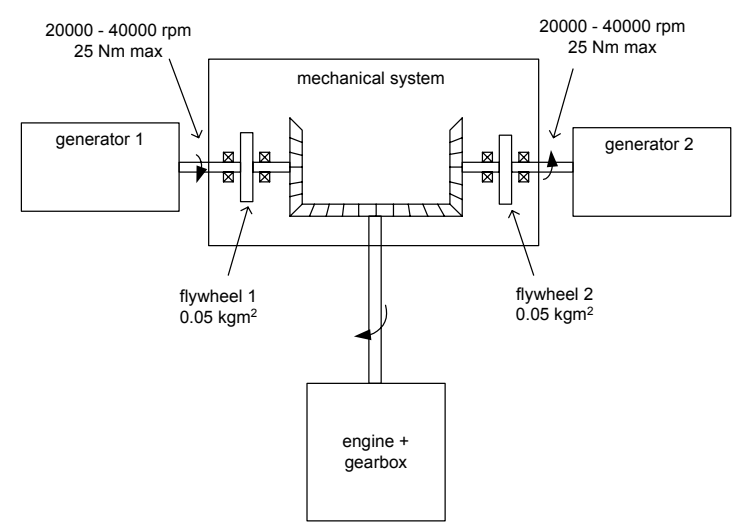

Fig. 1 - Schéma de principe du générateur inertiel.

\section{DESCRIPTION DE LA BOITE MECANIQUE}

La société SNA AUVERLAND à SAINT GERMAIN LAVAL (France) est en charge de l'étude et la réalisation du multiplicateur, les engrenages coniques et les volants d'inertie. Ceuxci sont localisés à l'extérieur des générateurs afin de simplifier leur construction et chaque volant est monté sur un arbre équipé de deux roulements.

Dans le cas du générateur $400 \mathrm{~kW}$ la masse de chaque volant d'inertie est de $22 \mathrm{~kg}$ et dans le cas du démonstrateur elle vaut $8 \mathrm{~kg}$. La vue $3 \mathrm{D}$ de la fig. 2 montre la boîte mécanique telle qu'elle sera réalisée, équipée des deux alternateurs :

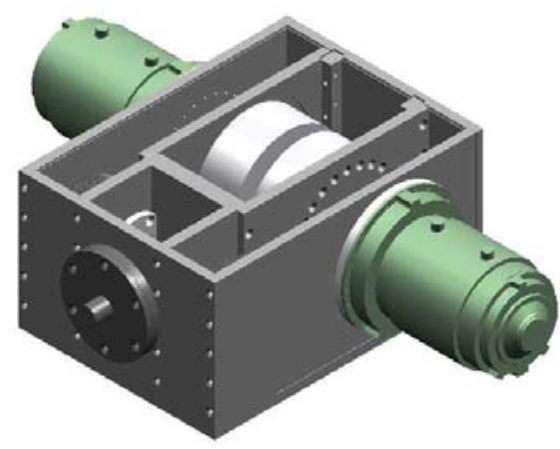

Fig. 2 - Vue de la boîte mécanique équipée des deux alternateurs.

\section{DESCRIPTION DU GENERATEUR}

Trois types de machine peuvent être envisagés pour ce type d'application :

La machine synchrone à aimants permanents,

La machine à réluctance variable et commutée,

La machine synchrone homopolaire à inducteur fixe.

Le choix a été effectué pour la machine $400 \mathrm{~kW}$ et le type de machine retenu a été appliqué au démonstrateur à réaliser.

Le générateur synchrone à aimants délivre un système polyphasé de tensions alternatives dont la fréquence dépend de la vitesse et dont l'amplitude dépend de la vitesse et de la charge. Comme dans cette application le générateur doit délivrer une tension continue régulée à $540 \mathrm{Vcc}$, il est nécessaire de prévoir, en sortie de l'alternateur, un convertisseur statique de puissance qui délivre une tension continue régulée. On peut concevoir ce convertisseur comme étant l'association d'un pont redresseur à diodes et d'un hacheur abaisseur. A ce niveau de puissance (200 KW par alternateur), un tel hacheur paraît encombrant lorsqu'il doit être monté sur un véhicule. D'autre part, les transistors devront supporter une tension voisine de $1500 \mathrm{~V}$, compte tenu de la variation de vitesse et de la variation de charge. Des transistors $1500 \mathrm{~V}-400 \mathrm{~A}$ existent mais demandent des dispositifs d'évacuation des pertes perfectionnés.

Le générateur à réluctance variable doit être lui aussi associé à un convertisseur statique à transistors de puissance. Mais, dans le cas de la machine à réluctance variable, le convertisseur est direct. Sa commande reste néanmoins encore délicate, surtout à ce niveau de puissance.

La machine synchrone homopolaire est composée de deux demi machines séparées par une bobine inductrice centrale, dont l'axe est confondu avec l'axe de rotation. Chaque demi machine est homopolaire, c'est à dire qu'elle ne comporte que des pôles de même nom, les pôles d'une demi machine étant opposés aux pôles de l'autre demi machine. Le flux magnétique, créé par la bobine inductrice, traverse successivement le premier entrefer, un pôle nord du rotor, l'arbre central du rotor, un pôle sud du rotor, le deuxième entrefer, un demi stator, la carcasse magnétique et enfin l'autre demi stator. Du fait que les pôles de nom opposé sont séparés dans le sens axial, la machine homopolaire est plus lourde qu'une machine synchrone classique, à vitesse égale. Cependant sa vitesse de rotation peut être bien plus élevée que celle d'une machine synchrone à rotor bobiné ou même que celle d'une machine synchrone à aimants permanents. En effet, son rotor massif lui permet d'atteindre des vitesses périphériques de l'ordre de $300 \mathrm{~m} / \mathrm{s}$ sans aucune difficulté, contrairement aux deux autres types de machine.

D'autre part, sa bobine inductrice permet de réguler le niveau des tensions de sortie avec un faible niveau de puissance correspondant aux pertes Joule de cette bobine ( de l'ordre du KW ). Il suffit donc de connecter un simple pont redresseur à diodes en sortie des phases de l'alternateur pour obtenir une tension continue régulée. On économise 
ainsi le hacheur de puissance, nécessaire dans le cas de la machine à aimants, le régulateur associé à la bobine d'excitation étant d'une conception beaucoup plus simple que celle du hacheur de puissance.

Pour ces différentes raisons, on a retenu dans cette application la machine synchrone homopolaire. La fig. 3 montre une coupe du générateur réalisé pour le démonstrateur. Une étude par éléments finis 3D a permis de calculer les pertes par courant de Foucault dans le rotor massif [1].

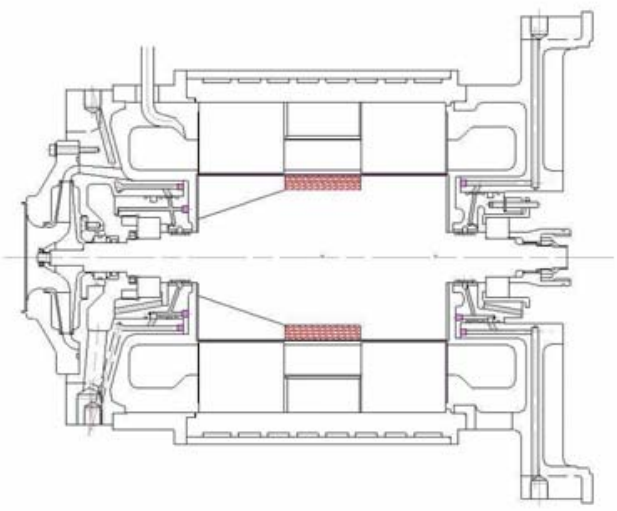

Fig. 3 - Coupe de l'alternateur démonstrateur.

Le rotor de la machine est monté sur deux roulements à billes à gorge profonde. Ils sont lubrifiés à l'huile. La carcasse extérieure possède des canaux intégrés dans lesquels de l'huile peut circuler pour refroidir le stator de la machine. Deux innovations ont été réalisées sur le démonstrateur :

- Un ventilateur a été étudié et réalisé par la société AIRTECHNOLOGIES à TOULOUSE (France). Monté en bout d'arbre, il permet d'évacuer efficacement les pertes aérodynamiques dans l'entrefer et les pertes Joule dans les développantes de bobinage. Le ventilateur a été étudié pour une vitesse de rotation supérieure à $50000 \mathrm{tr} / \mathrm{min}$.

- Le rotor possède des pôles saillants qui créent des pertes aérodynamiques importantes à $40000 \mathrm{tr} / \mathrm{min}$. Un carénage du rotor a donc été réalisé à l'aide d'une chemise cylindrique en fibre de carbone.

La masse de chaque alternateur du démonstrateur est égale à $61 \mathrm{~kg}$. Les photos des fig. 4 et 5 montrent le stator bobiné et le rotor de l'un des alternateurs.

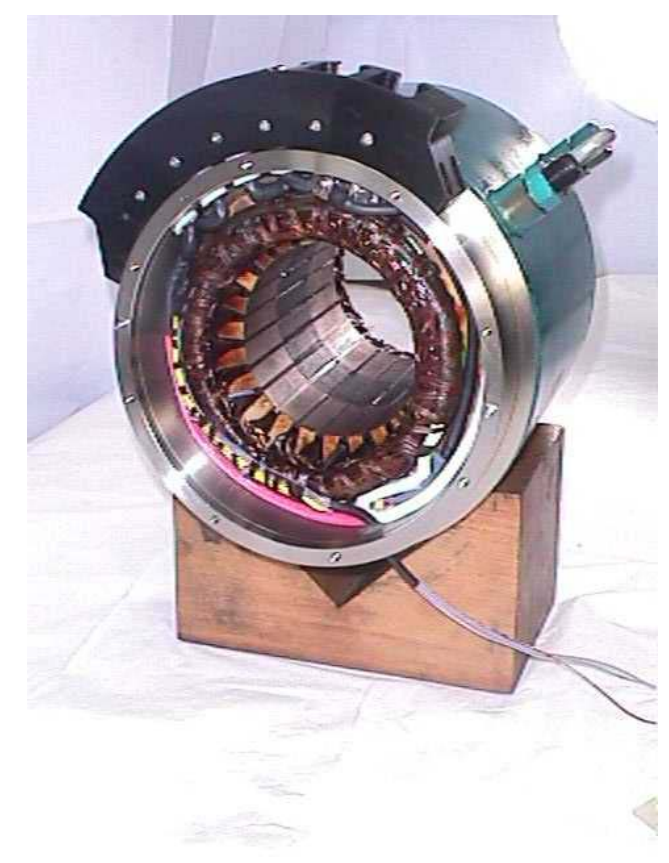

Fig. 4 - Stator bobiné

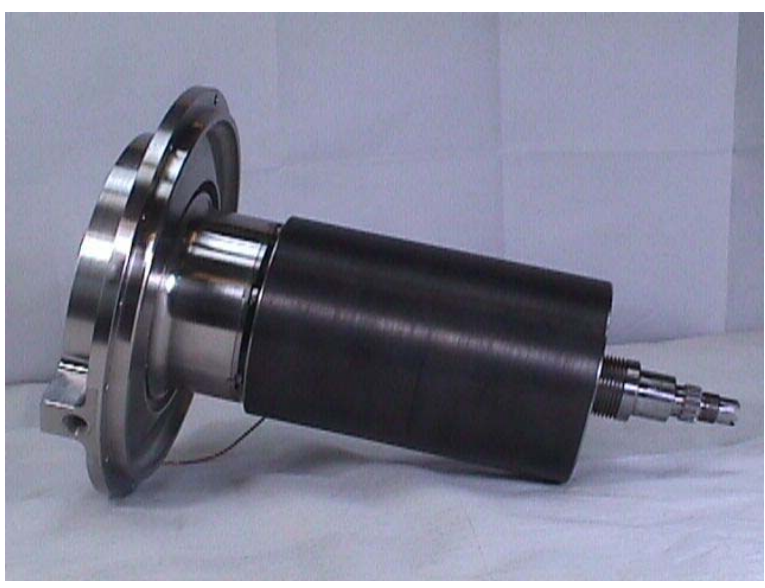

Fig. 5 - Rotor fretté en fibre de carbone

\section{DESCRIPTION DU GENERATOR CONTROL UNIT (GCU)}

Le GCU a deux fonctions distinctes :

1. Redresser le système triphasé de tensions délivré par chaque alternateur au moyen d'un pont de diodes,

2. Réguler le courant continu redressé délivré par chaque alternateur, via le pont redresseur, en agissant sur le courant inducteur de leur bobine d'excitation.

Les deux alternateurs sont connectés en parallèle via leur pont redresseur. La batterie 540 VDC du véhicule est elle même connectée en parallèle sur le réseau continu. La batterie impose donc la tension 
aux bornes des ponts.

Le GCU est constitué de deux canaux identiques correspondants aux deux alternateurs. Chaque canal est constitué d'un pont redresseur et d'un hacheur qui alimente la bobine d'excitation et dont l'entrée est connectée à la sortie du pont redresseur. La puissance d'excitation est au maximum égale à $1.5 \mathrm{~kW}$.



Pour chacun des alternateurs, la régulation est assurée par deux boucles mises en cascade. La boucle interne, la plus rapide, contrôle le courant $\left(\mathrm{I}_{\mathrm{EX}}\right)$ dans la bobine d'excitation de l'alternateur. La boucle externe contrôle le courant principal $\left(I_{R}\right)$ en sortie de redresseur, elle fournit à la boucle interne la consigne de courant d'excitation.

Le superviseur système du véhicule délivre une consigne de courant. Cette consigne est envoyée aux deux régulateurs de telle sorte que chaque générateur fournisse la moitié du courant demandé.

Dans l'optique d'évaluer les régulations de nos alternateurs, sur différentes technologies, la régulation de chaque canal a été choisie entièrement numérique. Elle est implantée sur une carte de commande construite autour d'un circuit de logique programmable FPGA de type 10K100 d'ALTERA.

Le codage des algorithmes de régulation est réalisé en VHDL et respecte une méthodologie de développement rigoureuse [2] avec comme objectif la constitution d'une bibliothèque de modules orientée «contrôle et régulation » et la possibilité de la réutiliser pour des développements futurs [3].



Fig. 7 - Procédure de développement sur cible FPGA

Les éléments de base, modules de la bibliothèque, permettent de reproduire les structures de régulation réalisées en simulation MATLAB/SIMULINK et donc de comparer les résultats de la simulation de la chaîne ALTERA aux résultats expérimentaux.

Les modules, y compris celui de plus haut niveau, répondent à la nomenclature de la figure 8 . Les signaux de contrôle des modules sont les suivants:

- Start : lancement des calculs (à la fréquence d'échantillonnage $20 \mathrm{kHz}$ ),

- Reset : signal asynchrone de retour à l'état 0 ,

- Init_int ; autorisation pour un nouveau calcul.



Fig. 8 - Modèle des modules.

Ces signaux sont générés par un séquenceur global qui organise la séquence des calculs au niveau le plus haut.

- L'horloge (clk) est une horloge haute fréquence $(10 \mathrm{MHz})$ qui cadence les calculs internes. Elle est commune à tous les modules. Issue du séquenceur global, elle synchronise les transitions des machines d'états.

- Le signal reset, commun à tous les modules, est utilisé notamment pour réinitialiser les registres des calculs (intégration,..) 
- Le signal init_int, commun à tous les modules, autorise le passage à l'état initial des machines d'états de tous les modules à la fin du calcul général.

Chaque module possède son propre séquenceur (machine d'états) qui organise les séquences des calculs internes et signale leur fin.

De la même façon au niveau hiérarchique le plus haut, le séquenceur global cadence, la propagation des données, après calcul, d'un module à l'autre, les signaux de fin de chaque module autorisant le passage au suivant.

La fig. 9 montre la chaîne simplifiée des calculs implantés dans le FPGA.

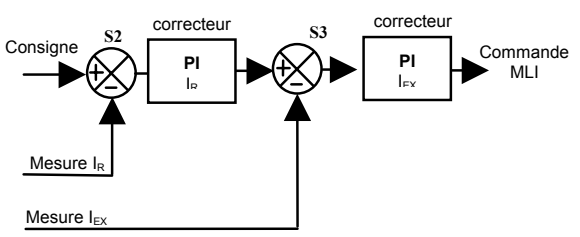

Fig.9 - Chaîne simplifiée des calculs.

Après acquisition des entrées mesures et consigne, l'ordre des calcul est le suivant :

1. Calcul du module « somme $\mathrm{S} 2$ »,

2. Calcul du module « réponse du Correcteur PI $\mathrm{I}_{\mathrm{R}} »$,

3. Calcul du module « somme S3 »,

4. Calcul du module « réponse du Correcteur PI $\mathrm{I}_{\mathrm{EX}} »$

Illustration par les résultats obtenus avec la boucle de régulation du courant d'excitation :

la figure 10 ci-dessous fait apparaître l'évolution du courant aux bornes de la charge RL (excitation alternateur) en réponse à une consigne en échelon de $3 \mathrm{~A}$.



Fig. 10 - Réponse indicielle du modèle numérique binaire et expérimentale

\section{RESULTATS DES ESSAIS EFFECTUES SUR LES} DEUX GENERATEURS

Les deux alternateurs et le GCU ont été testés sur banc d'essais THALES AES. Les fig. 11 et 12 montrent une machine sur le banc et l'armoire électronique prototype contenant le GCU global.



Fig. 11 - Machine sur banc d'essais

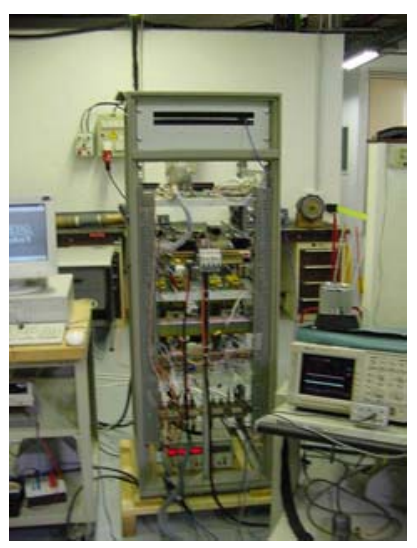

Fig. 12 - GCU pendant les essais

Les alternateurs ont été entraînés jusqu'à 50000 tr/min. Le niveau vibratoire mesuré sur la carcasse des machines est resté faible jusqu'à cette vitesse. Les comportements mécaniques du ventilateur et de la chemise en carbone du rotor sont donc satisfaisants.

Le débit mesuré du ventilateur est conforme aux attentes : $60 \mathrm{~g} / \mathrm{s}$ à $40000 \mathrm{tr} / \mathrm{min}$. On a pu mesuré aussi la puissance mécanique absorbée par le ventilateur : $1.7 \mathrm{~kW}$ à $40000 \mathrm{tr} / \mathrm{min}$.

Les essais en charge ont montré que la machine est suffisamment refroidie par le ventilateur intégré pour que la circulation d'huile initialement prévue dans la carcasse puisse être supprimée. Le courant d'excitation est conforme aux prévisions à la charge maximum $50 \mathrm{~kW}: 5.2 \mathrm{~A}$ à $40000 \mathrm{tr} / \mathrm{min}$ et $8 \mathrm{~A}$ à 20000 tr :min.

Des essais de transitoires de charge et de transitoires de vitesse ont été réalisés afin de tester la régulation de courant du GCU. Les fig. 13 et 14 montrent ces transitoires. Les transitoires de charges montrent que seul le courant continu est 
régulé tandis que la tension continue varie du fait que aucune batterie 540 VDC n'était connectée en parallèle sur les ponts redresseurs durant les essais sur le banc THALES.

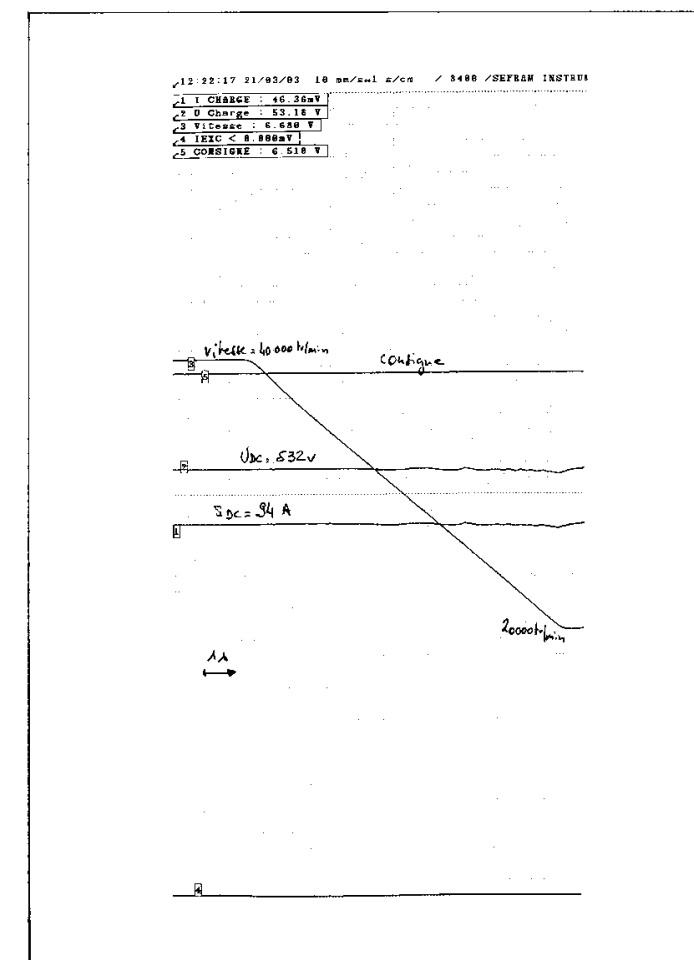

Fig. 13 - Régulation de courant à 94 ADC pendant une décroissance de vitesse 40000 - $20000 \mathrm{tr} / \mathrm{min}$ de durée $10 \mathrm{sec}$

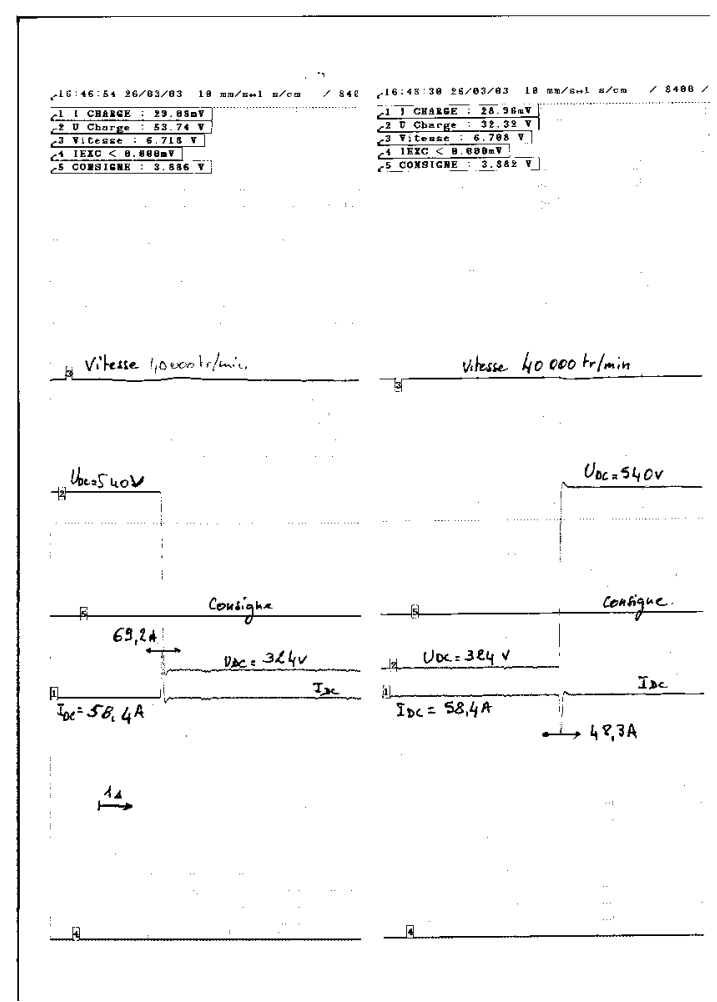

Fig. 14 - Transitoires de charge à $40000 \mathrm{tr} / \mathrm{min}$ (retrait de charge à gauche et application de charge à droit)

\section{CONCLUSION}

Un démonstrateur de générateur inertiel capable de délivrer une forte surcharge en prélevant la puissance de l'énergie cinétique emmagasinée dans un volant d'inertie a été étudié et réalisé. Sa structure permet de réduire le couple gyroscopique exercée par ce volant sur le véhicule. Les deux alternateurs qui le composent sont de type synchrone homopolaire. Les essais des générateurs ont montré la robustesse de leur rotor à 40000 tr/min. Le GCU qui redresse les tensions alternatives délivrées par les alternateurs et qui régule numériquement le courant continu de sortie a rapidement bien fonctionné.

Les inerties tournantes seront prochainement essayées avec le moteur thermique d'entrainement, les générateurs et le GCU sur une plate-forme roulante. $\mathrm{Si}$ le générateur démonstrateur fonctionnent correctement durant ces essais, il pourra être extrapolé à un générateur inertiel de 400 $\mathrm{kW}$ pouvant être embarqué sur un véhicule de combat électrique.

\section{BIBLIOGRAPHIE}

[1] N.BOUKARI, « Modélisation du mouvement à l'aide de codes de calcul par éléments finis en 3D : application à la machine homopolaire et au microactionneur électrostatique », Thèse de docteur de l'Institut national Polytechnique de Toulouse Spécialité génie Electrique Juil 2000.

[2] S.EUDIER, «Evaluation des possibilités d'utiliser des FPGAs pour réaliser une commande numérique de moteur », stage de fin d'étude IUP GEII Cergy Pontoise. Juil 2000.

[3] R.DCHIMI, « Etude et synthèse d'un correcteur numérique à base de FPGA » stage de fin d'étude IUP GEII Cergy Pontoise. Juil.2002. 